\title{
Protokoll der Mitgliederversammlung vom 4. September 2020 in Leipzig
}

Beginn: $16.30 \mathrm{Uhr}$

Ende: $18.00 \mathrm{Uhr}$

Teilnehmer: 64

Der Präsident, Prof. Markus Stücker, begrüßt die Teilnehmer und stellt fest, dass die Versammlung mit Veröffentlichung der Tagesordnung im Heft 4/2020 der Zeitschrift Phlebologie satzungsgemäß einberufen wurde.

Die Teilnehmer der Mitgliederversammlung erheben sich für eine Schweigeminute zu Ehren der seit der letzten Mitgliederversammlung verstorbenen Mitglieder.

\section{Genehmigung der Tagesordnung}

Die Tagesordnung wird angenommen.

\section{Genehmigung des Protokolls der Mitgliederversammlung vom 20.09.2019 in Münster (veröffentlicht in Phlebologie 6/2019)}

Das Protokoll wird einstimmig angenommen.

\section{Bericht des Präsidenten}

Insgesamt ist die Mitgliederentwicklung der DGP nach wie vor erfreulich, mittlerweile zählt die DGP knapp über 1800 Mitglieder.

Ca. die Hälfte der Mitglieder ist chirurgisch geprägt. Auch die Zahl der Allgemeinmediziner ist konstant hoch.

\section{Wissenschaftspreise 2020}

Insgesamt gibt es folgende Wissenschaftspreise, die in Kooperation mit der DGP verliehen werden:

- Sigvaris-Förderpreis Phlebologie

- Dr. med. André Janssen, Lübeck (5000€ für phlebologische Kongresse und Fortbildungen mit paralleler Berichterstattung)

- Bauerfeind-Doktorandenprogramm

- André Janssen, Lübeck: Venöse Hämodynamik der Femoralgefäße im Wasser im Vergleich zur klassischen Kompressionstherapie (betreut durch Prof. Kahle)

- Sophie Kindermann, Greifswald: Nebenwirkungen und Tragekomfort von Kompressionstrümpfen bei Personen mit orthostatisch bedingter Missempfindung und Ödembildung aufgrund sitzender Tätigkeit (betreut durch Prof. Dr. M. Jünger)

- Juzo-Forschungspreis Phlebologie

- Dr. Greta Zinser, Lübeck (Forschungsvorhaben: Kompression bei der Behandlung des Erysipels mittels medizinisch adaptiven Kompressionssystemen - KEMAKStudie)

- Kreussler Travel Awards (wurden aus den eingereichten Abstracts ausgesucht)

- Dr. M. Schiffmann, Münster, für ihre Arbeit Erfolgreiche Sklerotherapie bei postthrombotischem Syndrom unter Therapie mit Rivaroxaban

- Dr. C. Möllenhoff für seine Arbeit Sclerotherapy concludes surgery sCOS-Technik; eine alternative Behandlungstechnik in der Therapie der symptomatischen Varikose bei Risikopatienten
- Várady-Vortragspreis

- J. Kalemba, Izbicko (PL: Miniphlebectomy according to Varady as a mother of aesthetic phlebology)

- Medi-Reisestipendien

- 15 Preise à $500 €$

Prof. Stücker erinnert hier v. a. die in Klinik tätigen Kollegen, ihre Doktoranden/Assistenten auf die Preise hinzuweisen und diese Preise aktiv zu bewerben!

Jahrestagungen

2019 Münster

Die Jahrestagung in Münster war sowohl wissenschaftlich als auch wirtschaftlich ein voller Erfolg. 1020 Teilnehmer, 123 Teilnehmer am Crashkurs Phlebologie, 155 Teilnehmer am Update Phlebologie, ein ausgebuchtes Weiterbildungsforum mit vielen jungen Gesichtern, ein ausgebuchter Vertiefungskurs der Phlebologie-Assistenten, 44 ausstellende Firmen - Zahlen, die für sich sprechen.

Ein herzlicher Dank geht einmal mehr an Prof. Dr. Tobias Görge für die tolle und intensive Arbeit!

Insgesamt pendeln sich die Teilnehmerzahlen auf den Jahrestagungen bei ca. 1000 Teilnehmern ein. Um dies zu steigern, sollte zukünftig versucht werden, v. a. Teilnehmer aus dem internationalen Bereich sowie dem Bereich der Hausärzte und der Pflege zu rekrutieren. Für Leipzig war Dr. Hirsch hier gerade bei der Internationalität auf einem sehr erfolgreichen Weg!

\section{Welt-Thrombose-Tag}

Der Welt-Thrombose-Tag fand am 11. Oktober 2019 in der Deutschen Parlamentari- 


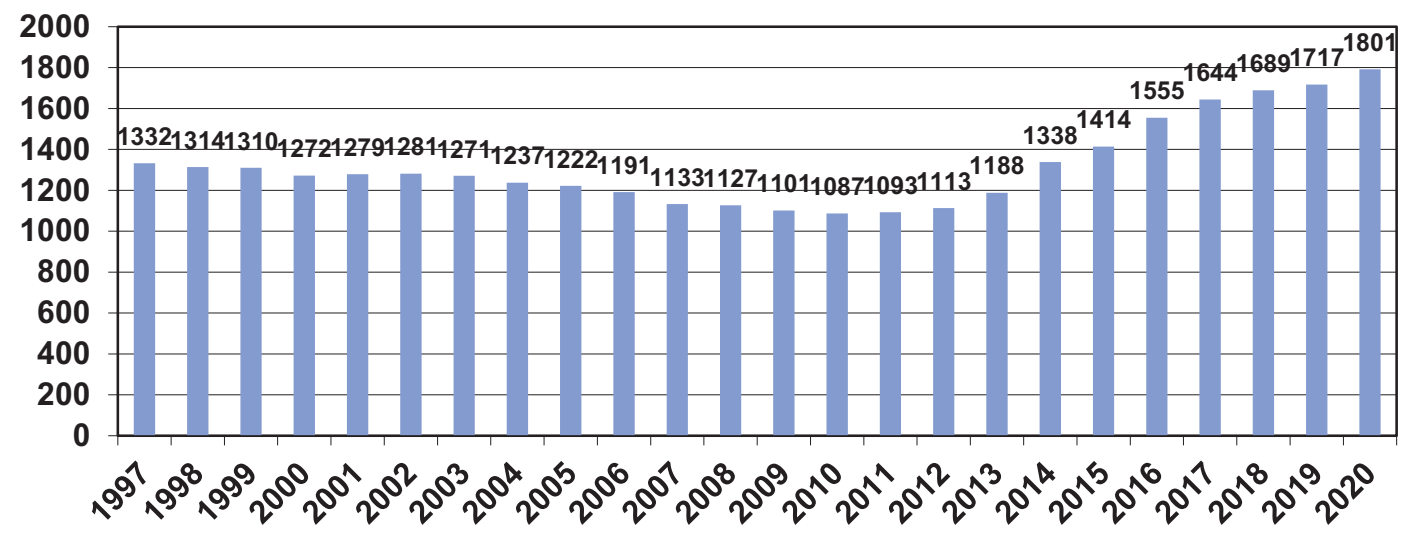

Entwicklung der Mitgliederzahlen der Dt. Gesellschaft für Phlebologie.

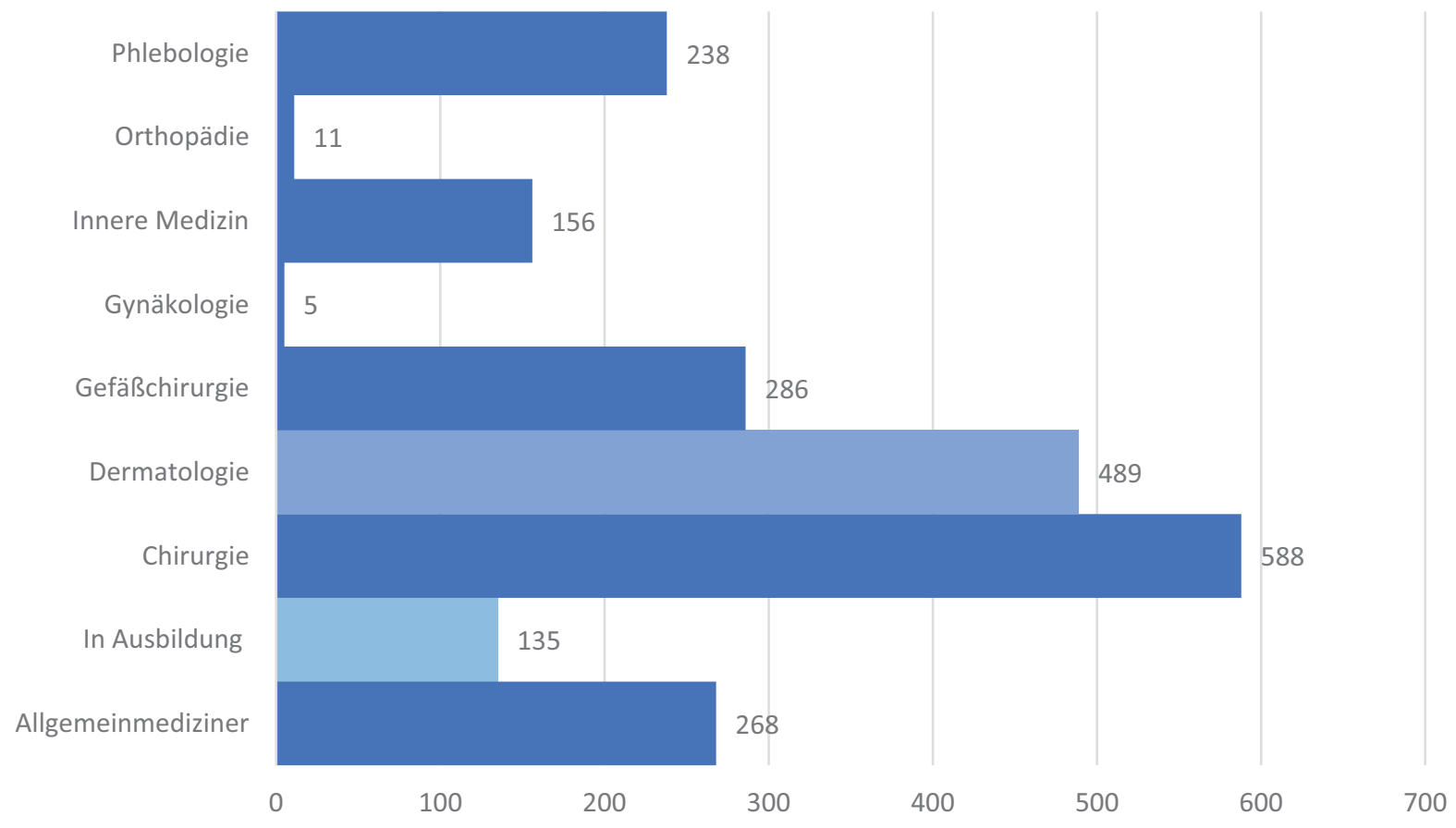

Verteilung der Mitglieder auf die verschiedenen Fachgruppen.

schen Gesellschaft in Berlin zum Thema „Alte Zöpfe abschneiden - Lungenembolie ambulant behandeln?" unter der Schirmherrschaft von Jens Spahn statt.

2019 wurde eine Publikation zum Thema Antiphospholipid-Positionsstatement eine Stellungnahme der wissenschaftlichen Fachgesellschaften GTH, DGP und DGA sowie von BDDH und Deutscher Gefäßliga und des Aktionsbündnisses Thrombose zum Rote-Hand-Brief vom 23.05.2019 verfasst. Darüber hinaus Newsletter und Facebook-Aktivitäten rund um den WeltThrombose-Tag.

Am 13.10.2020 findet der WTD zum Thema „Risiko Thrombose: 60 Jahre Pille - was sind die Fakten?" als Onlineveranstaltung statt.
Darüber hinaus hat das Aktionsbündnis Thrombose gemeinsam mit dem Bayrischen Gesundheitsministerium ein Versorgungsprojekt zum Thema „Bedarfsgerechte und qualitätsorientierte Versorgung von Patienten mit venöser Thrombose in Bayern (BEQUEST) * “ initiiert. 


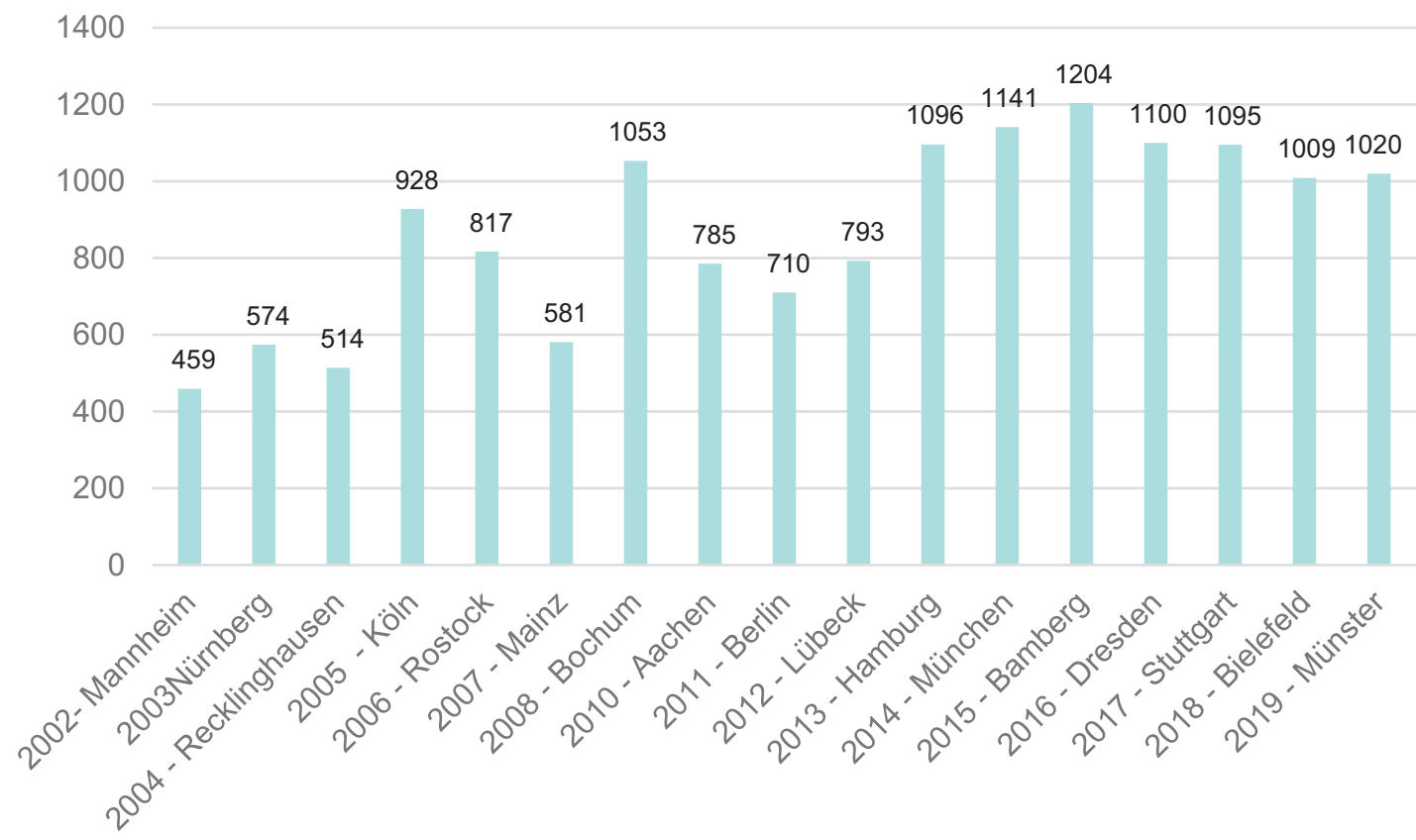

Entwicklung der Teilnehmerzahlen bei den Jahrestagungen der Dt. Gesellschaft für Phlebologie.

2020 ist auch die Deutsche Gesellschaft für Gefäßchirurgie und Gefäßmedizin dem Aktionsbündnis Thrombose beigetreten.

\section{Bericht der Generalsekretärin}

Frau Dr. Mendoza bedankt sich ausdrücklich persönlich bei Tobias Hirsch, der das Projekt Jahrestagung 2020 mit unvorstellbarem Nachdruck, liebevoll und über alle Hürden hinweg organisiert hat und „Neuigkeiten“, wie die Absage des Zoos, dann die gesamte COVID-Situation etc. in einer bewundernswerten Gelassenheit getragen hat.

Frau Dr. Mendoza bot in diesem Jahr im Rahmen des wissenschaftlichen Programms einen International-MasterclassUltraschallkurs an, der sehr gut angenommen wurde.

- Grundsätzlich machen derartige Kurse das Programm der Tagung sehr attraktiv.

- potenzielle Themen für weitere Masterclass-Kurse: Sklerotherapie, Wundbehandlung, endovenöse Therapie

Frau Dr. Mendoza berichtet mit Bedauern von der Verschiebung des EVF von 2022 auf 2023, da so die Abstracts nicht ohne weiteres parallel genutzt werden können.

Der Verkauf der Patientenflyer läuft nach wie vor sehr gut. Beide Flyer sind mittlerweile in der 2. Auflage. Ein Flyer zum Thema „Rezidivprophylaxe nach Thrombose“ ist in Vorbereitung.

Für die Nachrichten der DGP bittet sie um den Input der Mitglieder - wenn jemand etwas „Berichtenswertes“ hat, möge er es bitte an sie schicken.

\section{Bericht des Schatzmeisters}

Die finanzielle Situation der DGP hat sich Dank der erfolgreichen Jahrestagungen in Bielefeld und Münster positiv entwickelt. Das momentane Guthaben der DGP beläuft sich auf rund $200000 €$ einschließlich der Gelder der Arbeitsgemeinschaften. Die Auflistung der Ein- und Ausgaben der letzten 5 Jahre kann beim Schatzmeister angefragt werden.

\section{Webseite}

Die Website der DGP wird extrem gut angenommen.
Folgende Zugriffszahlen sind registriert:

- Mai 2020: 31489 Besuche

- Juni 2020: 36023 Besuche

- Juli 2020: 37853 Besuche

Die Arztsuche ist in den letzten 2 Monaten über 70000-mal aufgerufen worden, ca. $50 \%$ aller Seitenaufrufe im selben Zeitraum, $42 \%$ der Seitenbesuche begannen auf einer Seite im Abschnitt „Venenkrankheiten“, allen voran das Lipödem, 34\% Einstieg über die Arztsuche. $90 \%$ der Besuche fanden auf Seiten im Bereich „Patienten“ (inkl. Arztsuche) statt.

Es wird noch einmal daran erinnert, sich bei der Arztsuche zu registrieren, damit die jeweilige Praxis auch gefunden wird. Bitte registrieren Sie sich 1 . bei „Meine Daten“ und 2. bei „Meine Arztsuche“.

\section{Bericht der Kassenprüfer}

Herr Dr. Peter Neff berichtet über die Kassenprüfung und bescheinigt ordentliche Rechnungsführung. Die beantragte Entlastung des Vorstands erfolgt einstimmig bei 5 Enthaltungen. 


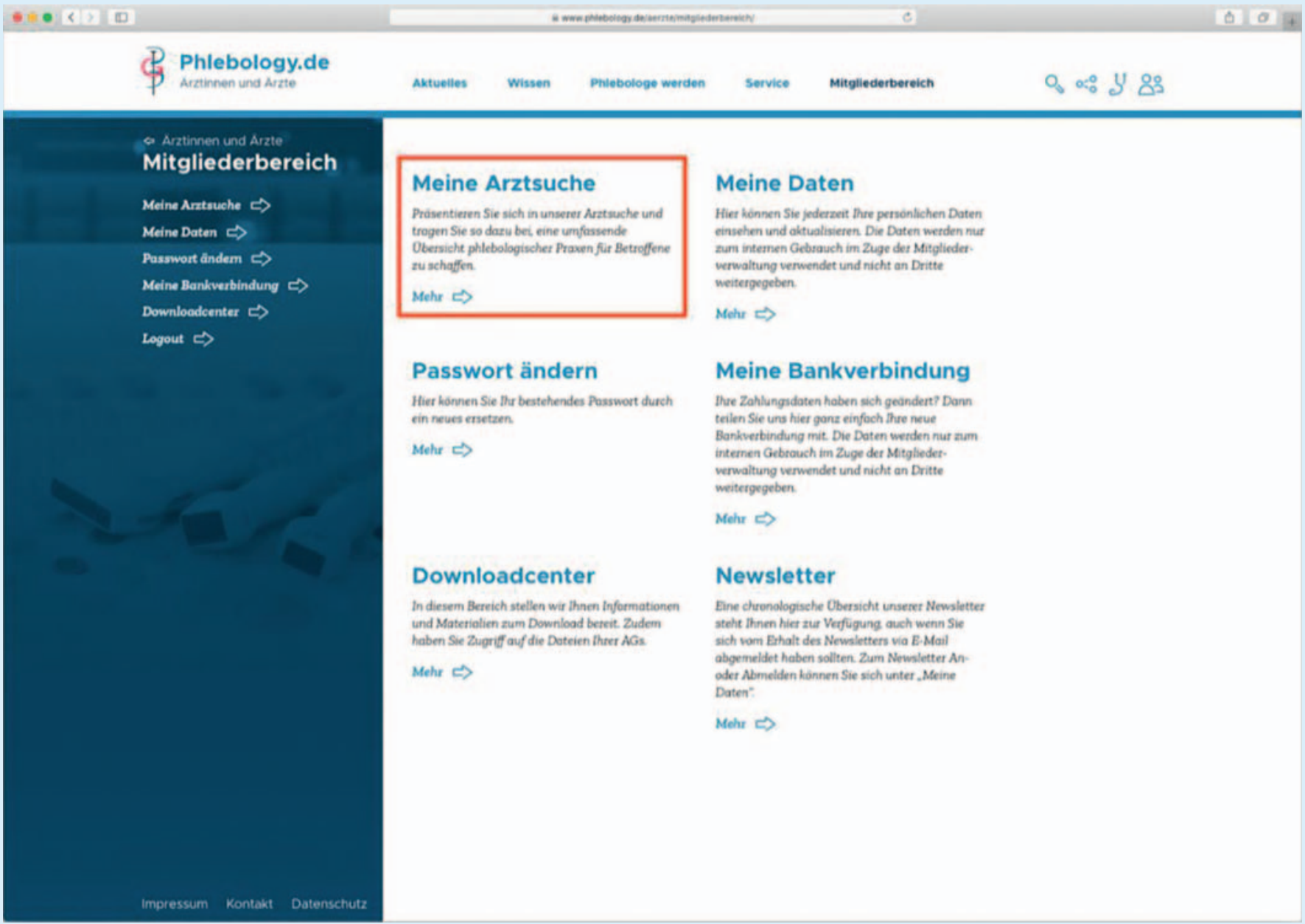

Zugang zur Maske für das Registrieren bei der Arztsuche auf der Webseite www.phlebology.de.

\section{Berichte der \\ Kongresspräsidenten}

\section{Leipzig}

Dr. Tobias Hirsch berichtet über die Coronabedingt sehr turbulente Vorbereitung der Tagung. Regelmäßig wurde abgewogen, ob die Veranstaltung stattfinden konnte. Die Internationalität, die vergangenes Jahr bereits angebahnt und von den Referenten zugesagt worden war, ist nun als ZoomHinzuschaltung dank der Streaming-Möglichkeiten garantiert. Er bedankt sich ausdrücklich bei wikonect für die technische Umsetzung des Projekts.

Corona-bedingt ist es insgesamt zu einer Reduktion der Sponsoringgelder gekommen, bei Mehrausgaben für Übersetzungen und dem Hybrid-Konzept. Nur ein Großsponsor (Bayer AG) hat sich komplett zu- rückgezogen, Sigvaris hat die Sponsorenleistungen halbiert.

Die Firma Medi hat hausintern entschieden, dass eine persönliche Teilnahme von MediMitarbeitern an Veranstaltungen 2020 nicht erlaubt ist (auch nicht bei der OTLeipzig). Medi ist aber als Hauptsponsor bei der Jahrestagung dabei, d. h. die Sponsoringzusagen werden eingehalten und die Standmiete wurde ebenfalls in Sponsoringgelder umgewandelt.

Insgesamt ein positives Signal: Eine Tagung auch zu Corona-Zeiten ist möglich!

\section{Aachen}

Die Vorbereitungen für die Jahrestagung in Aachen laufen „nach Plan“. Das Motto der Tagung lautet Aachen 2021 - Synergie und Vielfalt. Sie findet statt vom 8.-11.09.2021 im Eurogress. Die bewährten Kernelemente
Crashkurs Phlebologie, Update Phlebologie, Update Wundmanagement und Weiterbildungsforum werden beibehalten. Ein weiterer Schwerpunkt werden zahlreiche Hands-on-Kurse sein.

\section{Weiterbildungsordnung}

Die Musterweiterbildungsordnung, 2018 in Erfurt beschlossen, wurde von ersten Landesärztekammern übernommen, z. B. Westfalen Lippe: „Mindestanforderungen gemäß §11 WBO: Facharztanerkennung und zusätzlich Phlebologie gemäß Weiterbildungsinhalten unter Befugnis“ (also keine Zeitvorgabe, Betonung der Kenntnisse und Fähigkeiten, berufsbegleitend möglich).

De facto ist die berufsbegleitende Weiterbildung allerdings noch nicht final geklärt. Ein Gutachten des Justiziars von BVP und DGP schlägt vor, 3 denkbare Modelle be- 
63. Jahrestagung Der Deutschen

Gesellschaft für Phlebologie

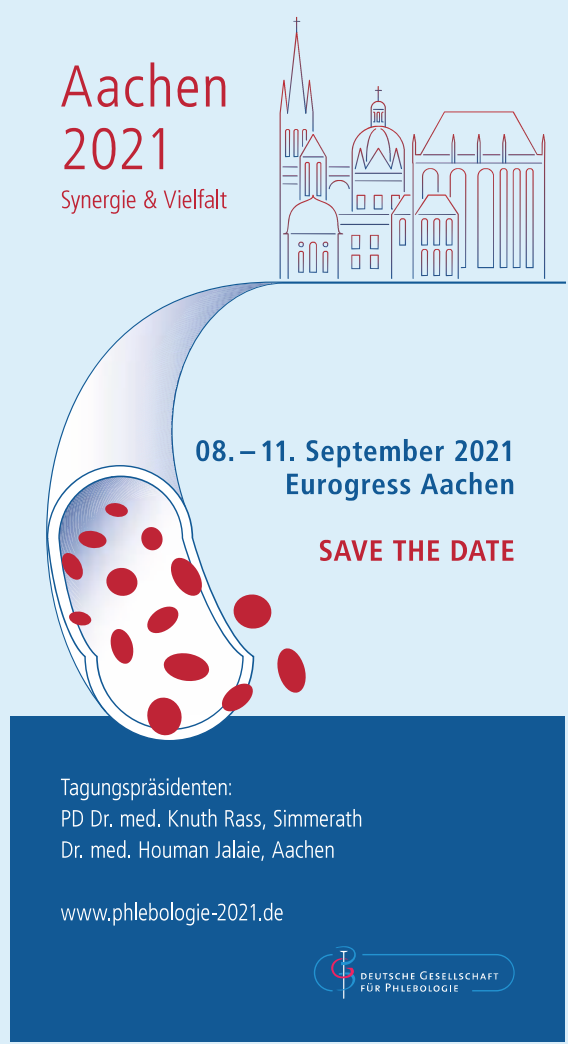

Ankündigung Jahrestagung DGP 2021 in Aachen.

rufsbegleitender Weiterbildung zu konkretisieren und mit der Bundesärztekammer vorabzustimmen. Dies sind:

- Weiterbildungspartnerschaft (überörtliche Gemeinschaftspraxis),

- Weiterbildung mit geringer wöchentlicher Tätigkeit (Kandidat geht stundenweise in die Praxis des Weiterbilders) und

- Consultant-Modell (Weiterbilder kommt in die Praxis des Kandidaten).

\section{Forschungsförderung und Forschungsbericht}

Es liegt ein Forschungsförderungsantrag von Prof. Kahle/A. Janssen zum Thema „Venöse Hämodynamik der Beine im Wasser" Synopsis VAFI-water Study vor. Ein positives Votum der Forschungsförderungskommission existiert ebenfalls. Der Antrag wurde im Rahmen der Vorstands- und Beiratssitzung der DGP positiv bewertet.
112 Artikel in MEDLINE-gelisteten Zeitschriften wurden von DGP-Mitgliedern veröffentlicht, 29 Veröffentlichungen fanden sich in der Phlebologie.

\section{Zusammenarbeit mit dem Berufsverband}

Der Berufsverband hat sich in diesem Jahr schwerpunktmäßig mit der Etablierung des Qualitätssigels VKZplus beschäftigt. Dr. Murena merkt an, ob es sinnvoll ist, in diesem Zusammenhang das „Phlebologicum" sozusagen durch die Hintertür mit ins Boot geholt zu haben. Es wird betont, dass das Phlebologicum nur die Plattform zur Dateneingabe bietet, die eigentlichen Daten dürfen wohl nicht genutzt werden.

Die GOÄ ist nach wie vor nicht fertig.

Den neuen EBM gibt es seit dem 1.04.2020. Keine wesentlichen Änderungen.

Es wurden zahlreiche Newsletter zu aktuellen berufspolitischen Themen verschickt.

\section{Leitlinien}

Folgende Leitlinien werden/wurden von der DGP bearbeitet:

- LL Sklerosierungstherapie abgeschlossen, Veröffentlichung Ende des Jahres

- LL Kompressionstherapie abgeschlossen, Veröffentlichung geplant

- LL Varikose - die Veröffentlichung der Leitlinie ist angesichts des Umfangs schwierig; hier werden Möglichkeiten eruiert

- LL Ulcus cruris - Koordinatorin Eva Valesky, Steuergruppe wird zusammengestellt

- LL Livedovaskulopathie S1 - Koordinator Tobias Görge, Steuergruppe arbeitet

- LL Lipödem S2k - Koordinatorin Gabriele Färber, Steuergruppe hat am 2.09.2020 getagt

\section{Fortbildungsakademie}

- Insgesamt gibt es 198 gültige und aktualisierte Fortbildungszertifikate der DGP.

- Relativ hohe Nachfrage im letzten Jahr auch bezüglich der Verlängerung - Auswirkungen der Kopplung VKZplus - FBZ.
- FBZ ist mittlerweile bei jedem Mitglied auch auf der Homepage hinterlegt mit Logo.

\section{Wie bekommt man das Fortbil- dungszertifikat Phlebologie?}

Ärzte mit anerkannter Weiterbildung Phlebologie:

- Mitgliedschaft in der DGP

- Nachweis von 20 Fortbildungspunkten im phlebologischen Bereich je Jahr in den letzten 3 Jahren

- Nachweis Zusatzbezeichnung Phlebologie

- Nachweis einer schwerpunktmäßigen phlebologischen Tätigkeit

Ärzte ohne anerkannte Weiterbildung Phlebologie, aber mit Facharztbezeichnung Dermatologie, Gefäßchirurgie oder Angiologie erwerben das Zertifikat

- Mitgliedschaft in der DGP

- Nachweis von 20 Fortbildungspunkten im phlebologischen Bereich je Jahr in den letzten 3 Jahren

- Nachweis einer schwerpunktmäßigen phlebologischen Tätigkeit

- Ablegen eines Kolloquiums zu phlebologischen Inhalten

Ärzte ohne abgeschlossene Weiterbildung Phlebologie und ohne Facharztbezeichnung Dermatologie, Gefäßchirurgie oder Angiologie

- Mitgliedschaft in der DGP

- Nachweis von 20 Fortbildungspunkten im phlebologischen Bereich je Jahr in den letzten 3 Jahren

- Nachweis einer schwerpunktmäßigen phlebologischen Tätigkeit

- Teilnahme am Grundkurs Phlebologie und an mindestens 2 von der DGP anerkannten Hands-on-Workshops

- Ablegen eines Kolloquiums zu phlebologischen Inhalten

- Der Vorstand der Fortbildungsakademie besteht aus 3 Personen, davon jeweils der amtierende Präsident der DGP und des BVP.

- 3. Person im Vorstand ist derzeit E. Rabe neben $\mathrm{H}$. Gerlach und M. Stücker.

- Der Beirat besteht aus 7 Personen:

K. Augustin, K. Hartmann, T. Goerge,

B. Kahle, D. Mühlberger, R. Murena und F. Pannier. 


\section{Fortbildungszertifikat Phlebologie}

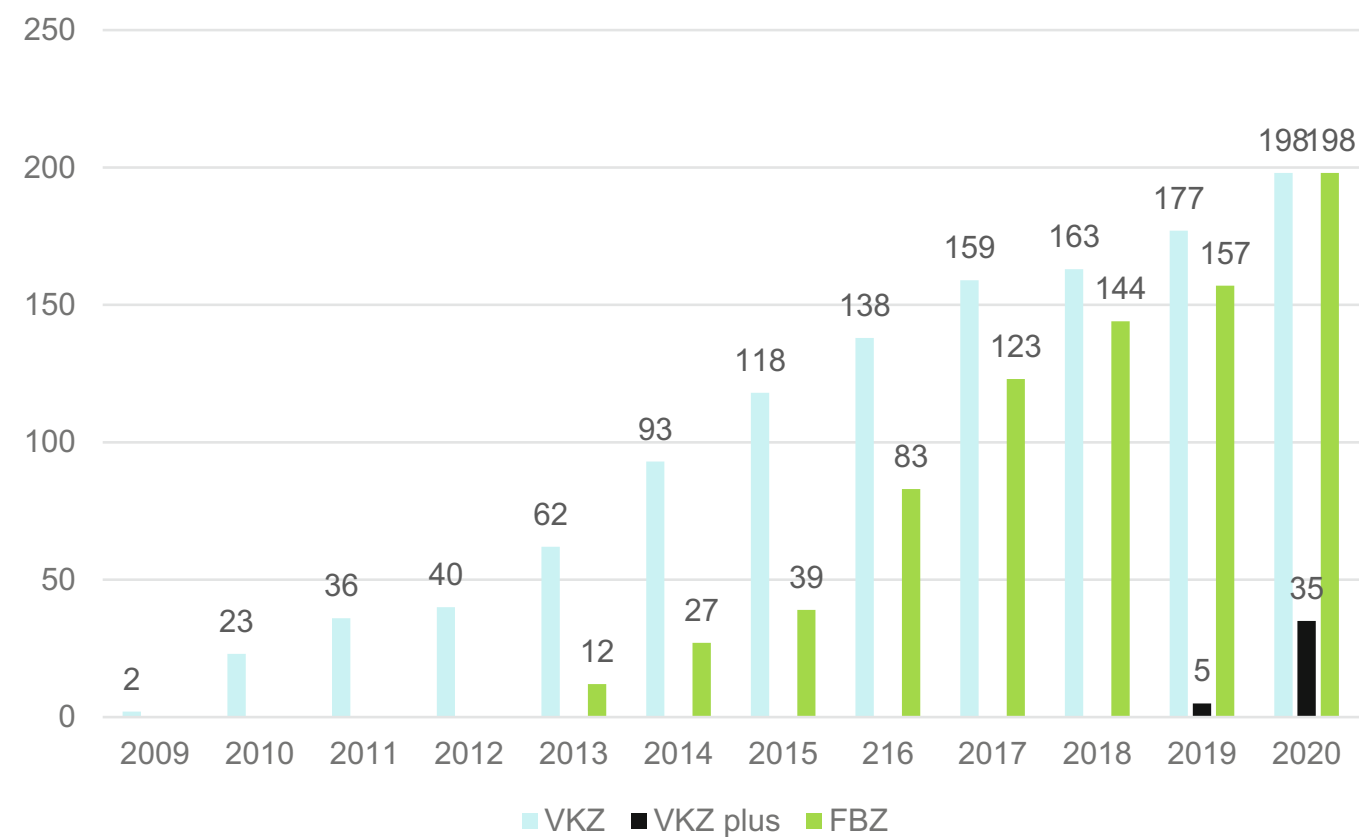

Entwicklung der Anzahl der Fortbildungszertifikate (grün), Venenkompetenzzentren (blau) und der Venenkompetenzzentren plus (schwarz).

- Im Rahmen des modularen Fortbildungskonzeptes werden über die FBA folgende Kurse angeboten:

- Sklero-Kurse (Bonn, Halle, Lübeck, Tegernsee)

- Mikrosklero-Kurse (München, Köln)

- Duplex-Kurse (Wunstorf)

- Grundkurs (Göttingen/Karlsruhe)

- Endovenös-thermische ablative Kurse (Freiburg, Halle/Bonn)

- Darüber hinaus findet die Ausbildung zum Phlebologie-Assistenten statt (Grundkurs BVT, Vertiefungskurs Jahrestagung).

\section{Zeitschrift Phlebologie}

Die Zusammenarbeit mit dem Thieme-Verlag ist gut und reibungslos. Der Zustrom von nationalen und internationalen Beiträgen ist vergleichsweise positiv. Im Rahmen der Schriftleitersitzung wurde über die Möglichkeit einer Aufnahme in den Science Citation Index Expanded (SCIE) berichtet.
Eine Listung im SCIE wäre dann möglich, wenn die Artikel der Phlebologie deutlich häufiger in gelisteten internationalen Publikationen zitiert werden.

\section{Berichte aus den Arbeitsgemeinschaften}

Folgende Arbeitsgemeinschaften sind in der DGP im Augenblick registriert:

- AG Sklerotherapie (Dr. Breu/Dr. Guggenbichler/Dr. Murena/Prof. Kahle)

- Out-AG (Dr. Hermanns)

- Endo AG (Dr. Hartmann, PD Dr. Pannier),

- Studieninitiierung unter Federführung von Frau Dr. Fink aus Heidelberg

- VOPAG (Prof. Mumme/Dr. Mühlberger)

- Planung eines OP-Kurses in Bochum für 2020

- Durchführung des VOP-Kurses in Innsbruck 2020

- AG Hämodynamik (Dr. Mendoza)

- AG Arbeitsmedizin (Dr. Gallenkemper)
- AG Lymphologie (Dr. Anya Miller)

- AG Thrombose (Dr. Jutta Schimmelpfennig)

- Interaktives Chatpodium zum interdisziplinären Austausch rund um das Thema „Thrombose“, Anfrage konkreter Expertenmeinungen, Beratung der Laien- und Fachpresse

- Hochaktuelles Thema: COVID-19 Gerinnung - Thrombose

- Prof. Bauersachs und Frau Dr. Schimmelpfennig sind Mitglieder der Steuerungsgruppe des Aktionsbündnisses Thrombose

- Durchführung einer gemeinsamen Sitzung der AG Thrombose und des Aktionsbündnisses am 3.09., konzipiert als „Anti-Symposium“

- AG Venöse Malformationen (Prof. Berlien)

- AG Junge Phlebologie (Dr. WoitallaBruning, Dr. Recke)

- AG Phlebologie-Assistenten (Dr. Murena)

- AG Pflege (Prof. Jünger)

- AG Hilfsmittelversorgung (PD Dr. Valesky) 
Die Arbeitsgemeinschaften stehen allen Mitgliedern offen. Bei Interesse zur Mitarbeit wenden Sie sich bitte an die jeweiligen Ansprechpartner. Einige Arbeitsgemeinschaften bieten Downloads (z. B. Aufklärungsbögen, Abrechnungsbögen) für ihre Mitglieder an, die über den Downloadbereich der Website abgerufen werden können.

\section{Verschiedenes}

Prof. Stücker weist auf den diesjährigen Jahresbericht hin. Er dankt dem WPV-Verlag für sein Engagement, diesen auch in diesem für alle wirtschaftlich schwierigen Jahr zu realisieren.
Gez.

Prof. Dr. med. Markus Stücker

Präsident

Gez.

Dr. med. Erika Mendoza Generalsekretärin 